Pädiatrie und Pädologie 2013 · 48:5

DOI 10.1007/s00608-013-0098-y

๑) Springer-Verlag Wien 2013

\section{R. Kerbl}

Landeskrankenhaus Leoben-Eisenerz , Abteilung für Kinder und Jugendliche, Leoben

\title{
Spitzenmedizin für Kinder
}

\section{Liebe Leserinnen und Leser,}

vor Ihnen liegt das „Kongressheft“ zur 51. Jahrestagung der Österreichischen Gesellschaft für Kinder- und Jugendheilkunde (ÖGKJ), welche vom 26. 09. bis 29. 09. 2013 in Innsbruck stattfindet. Das Innsbrucker Team um Herrn Prof. Dr. Stein (Tagungspräsident) und Frau Prof. Dr. Kiechl-Kohlendorfer (Tagungssekretärin) hat ein spannendes und vielfältiges Programm zusammengestellt, welches sicher viele Interessierte nach Innsbruck bringen wird. Herr Professor Stein nimmt in diesem Heft zu den Schwerpunktthemen, den Intentionen und zum Ablauf der Innsbrucker Jahrestagung Stellung.

Im Rahmen der Innsbrucker Jahrestagung ist auch die Vorstellung eines gemeinsamen Projektes zwischen dem Autor und Geschichtenerzähler Folke Tegetthoff und der ÖGKJ vorgesehen. Es geht darum, Eltern zum Vorlesen und Geschichtenerzählen zu animieren, und damit der zunehmenden "Sprachlosigkeit“ unserer Gesellschaft zu begegnen.

Besonders hinweisen möchte ich auf den Beginn einer „Kleinserie“ zum Thema hochspezialisierte Medizin (HSM) / Schwerpunktbildung / Zentrenbildung. Diese Serie, die sich in den Folgeheften fortsetzen wird, geht zurück auf einige verdiente ÖGKJ-Mitglieder, welche in ihrem Ruhestand der österreichischen Pädiatrie noch ein paar Gedanken zur sinnvollen Entwicklung der "Spitzenmedizin“ mitgeben wollen.

Das österreichische Gesundheitswesen ist - wie jenes der meisten anderen entwickelten Länder - im Umbruch. Immer neuere, bessere, vor allem aber auch teurere Therapieoptionen machen das $\mathrm{Ge}$ sundheitswesen scheinbar nach und nach unfinanzierbar, weshalb Ausgabenlimitierungen, Rationalisierungen, teilweise aber auch Rationierungen nicht nur angedacht, sondern teilweise bereits umgesetzt werden. Die „Spitzenmedizin“ stellt dabei einen wesentlichen Kostenfaktor dar, weshalb sie sinnvollerweise auf einige wenige Zentren konzentriert werden muss. Letzteres ist nicht nur eine ökonomische Frage, sondern vor allem auch eine Frage der Versorgungsqualität.

Sensibel wird der Bereich „Schwerpunktsetzung“ bzw. „Zentrenbildung“, wenn überlegt wird, wo solche Zentren errichtet werden sollen und wer diese betreiben sollt. Es steht außer Frage, dass derartige „Zentren“ vor allem an universitären Einrichtungen sinnvoll sind. Manchmal stehen aber auch Partikularinteressen und individuelle Begehrlichkeiten im Vordergrund, die bei anderen durchaus auch Angst machen können. Allzu rasch kann unter dem Deckmantel "Qualitätssicherung“ eine solche Zentrenbildung auch zum „Patientenklau“ ausarten und die Pädiatrie in ihrer Gesamtheit „zerreißen“.

Die teilweise auf fragwürdigem Niveau geführte Diskussion in Deutschland um „Mindestzahlen“ kann für die österreichische Pädiatrie nicht wirklich als Vorbild gelten, und es ist wohl auch kein Zufall, dass die Schweizer Kantonsvereinbarung zur „Zentrenbildung“ sich letztlich auf einige wenige, vorwiegend sehr seltene Indikationen zurückzieht.

Und es kann auch nicht die Intention einer solchen Entwicklung sein, nichtuniversitäre Abteilungen zu „Kinderabteilungen für Schnupfen und Durchfall“ zu degradieren. Der Prozess der „Schwerpunktsetzung" erfordert daher eine sorgfältige Analyse, ein klares Ziel, v.a. aber ein patientenorientiertes Denken und ein kollegiales Miteinander aller Beteiligten.

Es gibt derzeit etwa 7.500 verschiedene „seltene Erkrankungen“ (orphan diseases).
Insgesamt machen die „seltenen Erkrankungen“ jedoch etwa 30 Prozent des pädiatrischen Krankengutes aus und stellen somit die größte Krankheitsgruppe dar. Damit ergibt sich für „Zentren“ durchaus auch die Verlockung, dieses Patientenkollektiv an sich zu ziehen. Dies kann in bestimmten Fällen von Vorteil sein, darf aber keinesfalls zur Abwertung der anderen Kinder- und Jugendabteilungen führen. Die Pädiatrie kann in ihrer Gesamtheit nur dann "gut" sein, wenn das Versorgungsniveau auf allen Ebenen "gut" ist.

Dabei muss immer die bestmögliche Versorgung im Sinne des Patienten im Vordergrund stehen. In vielen Fällen bedeutet das zentrale Steuerung und Datensammlung, aber soweit wie möglich dezentrale Versorgung. Derartige Versorgungskonzepte sind z.B. in den Bereichen Pädiatrische Hämato-Onkologie und Pädiatrische Rheumatologie gut gelungen, in anderen Bereichen (Bsp. „Intersex") aber bisher gescheitert.

Das Thema „Zentrenbildung“ IST bereits Diskussionspunkt der österreichischen Gesundheitspolitik. Die österreichische Pädiatrie sollte sich dieser Diskussion nicht verschließen, sondern sie auf patientenorientiertem und fairem Niveau aktiv führen, und damit eine sinnvolle Entwicklung (mit-)bestimmen.

Ich wünsche Ihnen viel Freude bei der Lektüre dieses „Kongressheftes“ und freue mich über jegliches Feedback zu den Inhalten.

Ihr

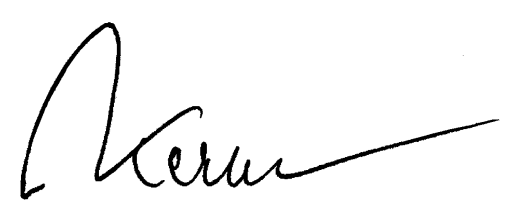

Reinhold Kerbl 